\title{
La diversidad beta: medio siglo de avances
}

\author{
Beta diversity: half a century of advances
}

\author{
Jaime M. Calderón-Patrón ${ }^{凶}$, Claudia E. Moreno e Iriana Zuria \\ Centro de Investigaciones Biológicas, Universidad Autónoma del Estado de Hidalgo. Apartado postal 69-1, 42001 Pachuca, Hidalgo, México. \\ \entropiajaime@yahoo.com
}

\begin{abstract}
Resumen. Se presenta una revisión de las principales tendencias que el estudio de la diversidad beta ha tenido a lo largo de más de 50 años, a partir de la propuesta del concepto y hasta las revisiones del tema realizadas recientemente. Es notable el incremento de publicaciones, en especial en la última década, donde se discuten y proponen sus métodos de análisis, aproximaciones de estudio, así como los conceptos y fenómenos biológicos relacionados. Este fructífero trabajo en la generación de ideas y conocimientos ha provocado un cambio importante en la conceptualización y en la forma de apreciar y analizar la diversidad beta, dando lugar a un marco de trabajo renovado y fresco, con un enorme potencial de aplicación en ecología, biogeografía y biología de la conservación.
\end{abstract}

Palabras clave: biodiversidad, comunidades, recambio de especies, composición de especies, similitud, anidamiento, diversidad verdadera.

\begin{abstract}
We review the main advances that the study of beta diversity has had for over 50 years, since its proposal and until the latest reviews. The increase in the number of publications is remarkable, especially in the last decade, and in these papers many authors discuss and propose methods for analysis, research approaches, as well as basic concepts and related biological phenomena. This fruitful task in the generation of ideas and knowledge has radically changed the ways in which beta diversity is conceptualized, appreciated, and analyzed, resulting in a renovated and fresh framework, with an enormous potential for application in ecology, biogeography, and conservation biology.
\end{abstract}

Key words: biodiversity, communities, species turnover, species composition, similarity, nestedness, true diversity.

Desde hace poco más de 50 años, cuando fue acuñado el término de diversidad beta $(\beta)$ como uno de los componentes de la diversidad de especies en una región (Whittaker, 1956, 1960), su conceptualización ha sido poco clara y se han generado diferentes definiciones y métodos para cuantificarla, mismos que hasta hace poco no habían sido evaluados apropiadamente (Jurasinski et al., 2009), lo que motivó que en la última década surgieran diferentes esfuerzos que han contribuido a comprender la diversidad $\beta$ (e.g. Vellend, 2001; Koleff et al., 2003a, 2003b; Legendre et al., 2005; Jost, 2006, 2007; Jurasinki et al., 2009; Moreno y Rodríguez, 2010, 2011; Tuomisto, 2010a, 2010b, 2010c, 2011; Anderson et al., 2011). Gracias a estas aportaciones, actualmente tenemos más claridad acerca de los distintos fenómenos biológicos que han sido relacionados y en ocasiones confundidos con la idea original de diversidad $\beta$. El concepto de diversidad $\beta$ también tiene gran relevancia en ecología y biogeografía para comprender, cuantificar y valorar la diversidad biológica, y puede considerarse como un concepto clave para entender el funcionamiento de los

Recibido: 06 junio 2011; aceptado: 16 marzo 2012 ecosistemas, para la conservación de la biodiversidad y para el manejo de los ecosistemas (Legendre et al., 2005).

El objetivo de este trabajo es dar a conocer las aproximaciones más sobresalientes relacionadas con la diversidad $\beta$, a estudiantes, investigadores y a un público no familiarizado con la literatura reciente sobre el tema. Por lo tanto, decidimos no incluir aquí los métodos de análisis utilizados para evaluar la diversidad $\beta$ y los fenómenos relacionados con ella, ni adoptamos ninguno de los conceptos ni propuestas metodológicas compiladas. Para los interesados, la antología más completa a la fecha de medidas de análisis se puede consultar en los trabajos de Tuomisto (2010a y 2010b) y Anderson (2011). El presente ensayo aborda los conceptos columnares de la diversidad $\beta$, y para ello se decidió dividir el trabajo en 3 etapas que muestran 1) el origen del concepto; 2) la expansión del concepto y la proliferación de medidas, y 3) los esfuerzos enfocados hacia una síntesis del concepto. Además, se exponen parte de las tendencias actuales del estudio de la diversidad $\beta$.

Etapa 1. El origen del concepto. El concepto de diversidad $\beta$ lo sugiere Robert $\mathrm{H}$. Whittaker por vez primera en 1956, sin darle un nombre en particular, al estudiar la variación 
en la composición de la vegetación en un gradiente altitudinal de la cordillera de las Great Smoky Mountains, en el sureste de los Estados Unidos de América (Whittaker, 1956). Sin embargo, el término diversidad $\beta$ fue acuñado en 1960 por el mismo Whittaker en el artículo "Vegetation of the Siskiyou Mountains, Oregon and California", donde analiza la diversidad de la flora de esa zona, encontrando que la riqueza de especies se incrementa desde la costa hacia el interior del continente (Whittaker, 1960). De acuerdo con Whittaker (1960), este cambio en la composición de especies es producto de la diversidad de sitios individuales y de la diferenciación relativa de los patrones de diversidad de la vegetación a lo largo de una mezcla de gradientes topográficos de humedad. Estos 2 factores motivaron en Whittaker (1960) la necesidad de distinguir 3 aspectos o niveles de la diversidad de especies en las comunidades naturales:

...1) la riqueza en especies de un sitio o comunidad particular, o de un estrato dado o grupo de organismos de un sitio (diversidad alfa $\alpha$ ); 2) la magnitud de cambio en la composición de las comunidades o el grado de diferenciación entre comunidades, en relación con un gradiente ambiental complejo o a patrones ambientales (diversidad beta, $\beta$ ); y 3) la diversidad de especies de un número de muestras o de comunidades para algún grupo de ambientes, los cuales están de tal forma combinados que el valor de diversidad (diversidad gamma, $\gamma$ ) es el resultado tanto de las diversidades $\alpha$ como de la diversidad $\beta$ de estas muestras.

La complejidad del concepto de diversidad $\beta$ ya la percibía Whittaker (1972), como lo demuestran sus siguientes frases:

Las diversidades $\beta$, en contraste con las diversidades $\alpha$ y $\gamma$, que son de un carácter dimensional diferente, están basadas en tasas o diferencias. Para diferentes propósitos de investigación dichas tasas o diferencias tienen que ser medidas a lo largo de clinas particulares o para un conjunto de muestras, difiriendo una de otra a lo largo de varios ejes de hábitat o del hiperespacio de la comunidad. Por lo tanto, diferentes aproximaciones son posibles para evaluar la diversidad $\beta$.

Esta complejidad conceptual llevó a Whittaker (1977) a clasificar los diferentes tipos de la diversidad de especies en 2 grandes grupos: diversidades de inventarios y diversidades de diferenciación. En el primero, coloca las diversidades $\alpha$ y $\gamma$, y en el segundo la diversidad $\beta$. Influenciado por aspectos relacionados con la diversidad $\beta$ propuestos por otros autores (e.g. MacArthur, 1965; Cody,
1975) y por la complejidad misma del concepto, Whittaker modifica su propia definición de diversidad $\beta$ (utilizando por primera vez el término de paisaje), y la define como "la magnitud de cambio en la composición de las especies a lo largo de un gradiente ambiental o entre diferentes comunidades en un paisaje" (Whittaker, 1977).

A la par de estas diferentes conceptualizaciones de la diversidad $\beta$, Whittaker sugirió varias formas de medirla: 1) la fórmula $\beta=\gamma / \alpha$, donde la diversidad $\beta$ es resultado de la diversidad $\gamma$ dividida entre la diversidad $\alpha$ promedio; 2) el porcentaje de similitud entre pares de muestras y 3) las unidades de cambio medio (half-change) de la similitud con la distancia (Whittaker, 1956). Un cambio medio se define como la distancia necesaria para reducir la similitud entre comunidades en un 50\% (Fig. 1).

Paralelamente, el ecólogo Robert H. MacArthur (1965) desarrolló ideas similares sobre el cambio en la composición de especies, describió un patrón de diversidad de especies dentro y entre hábitats con referencia a la teoría de biogeografía de islas, según la cual conforme una isla se va saturando de especies, éstas van restringiendo su hábitat poco a poco. Con este proceso, durante los estadios iniciales de colonización, la diversidad de especies será totalmente dentro de un hábitat, ya que los primeros colonizadores tendrán la posibilidad de ocupar toda la isla, y conforme más especies llegan, la distribución de las mismas se irá restringiendo de acuerdo con sus requerimientos fisiológi$\cos$, lo que provocará un incremento de la diversidad entre hábitats. MacArthur (1965) menciona además que en los trópicos existen más áreas compuestas por varios tipos de hábitats, con una mayor diversidad, que en las zonas templadas, y añade que eso se debe a una subdivisión más fina de los hábitats, más que a un incremento notable de la diversidad dentro de los hábitats. No obstante, MacArthur no desarrolló el concepto de diversidad entre hábitats con mayor detalle ni los métodos para su medición, por lo que sus ideas no trascendieron tanto como las de Whittaker en la consolidación del concepto de diversidad $\beta$ como tal.

Whittaker (1956 y 1960) propuso 2 conceptos relacionados con la diversidad $\beta$ que hoy siguen vigentes: el decaimiento de la similitud con la distancia (distance decay in similarity) y el recambio de especies (species turnover). Decaimiento de la similitud con la distancia. Este fenómeno se presenta cuando la similitud en la composición de especies entre 2 sitios disminuye conforme se incrementa la distancia entre ellos (Whittaker, 1956; Nekola y White, 1999). Este patrón está íntimamente relacionado con las unidades de cambio medio de la similitud con la distancia a lo largo de un gradiente ambiental (Fig. 1), ya que si la similitud disminuye con la distancia, el recambio de especies (explicado en la siguiente sección) aumenta con la distancia (Fig. 2). El análisis del patrón de disminución de 


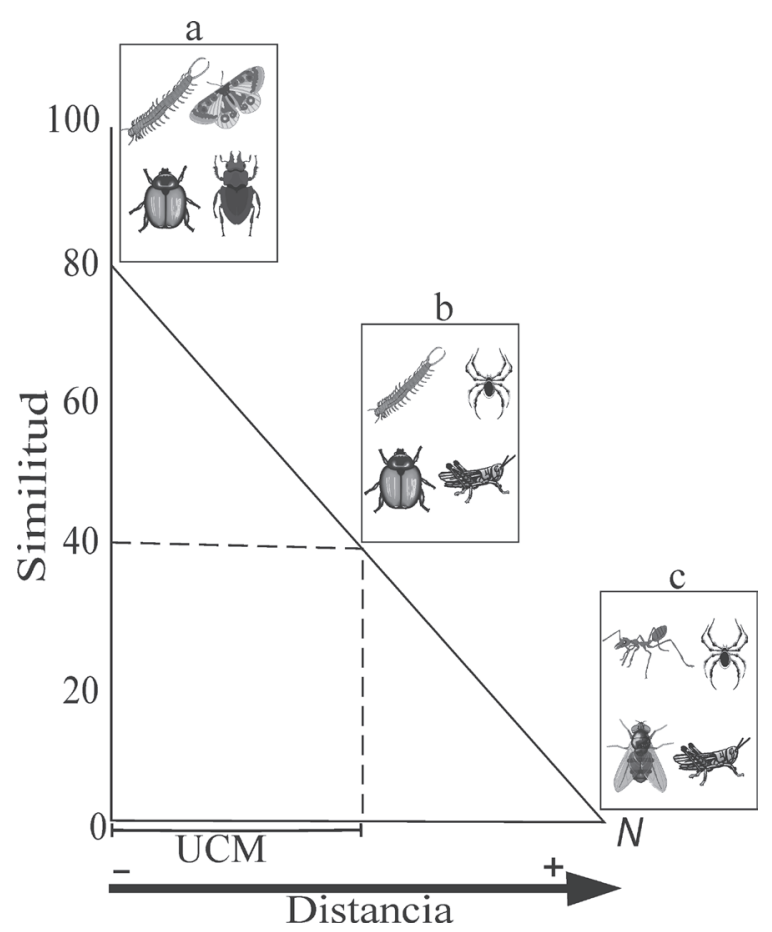

Figura 1. Medición de la diversidad beta con unidades de cambio medio. Comunidades hipotéticas (a, b, c) con una distancia de $N$. La similitud en la composición de especies disminuye conforme aumenta la distancia entre las comunidades. La distancia a la cual la similitud disminuye a la mitad (de 80 a $40 \%$ ) representa una unidad de cambio medio (UCM), lo que constituye una medida de diversidad beta (Whittaker, 1960).

la similitud con la distancia se puede realizar mediante un modelo linear o no linear, o mediante la tasa de recambio, que es la pendiente estimada de la relación entre la similitud y la distancia (Anderson et al., 2011). Uno de los pioneros en estudiar el decaimiento de la similitud con la distancia a diferentes escalas fue Preston (1960), quien observó que la tasa de recambio de la composición de especies de aves a través del espacio dentro de un continente, es menor que a través de los continentes. Preston, atribuye esta alta tasa de recambio a través de los continentes a la diversificación evolutiva (especiación) entre faunas como un resultado de limitación en la dispersión y a las bajas tasas de recambio de las especies de aves dentro de los continentes como un resultado de la variación ambiental (Preston, 1960).

Para explicar el patrón de decaimiento de la similitud con la distancia se han propuesto 3 mecanismos principales. El primer mecanismo por el cual este patrón podría presentarse son las condiciones ambientales que cambian conforme aumenta la distancia, es decir, hay una disminución de la similitud ambiental con la distancia, lo que implica que haya una separación de las especies con diferentes características fisiológicas, que con el paso del tiempo provocan que la composición de especies sea relati- vamente predecible de acuerdo con el tipo de ambiente. El proceso se ha explicado mediante el modelo de diferencia de nicho (Nekola y White, 1999; Soininen et al., 2007). El segundo depende de la configuración del ambiente (el contexto espacial y el tiempo), pues esta configuración influye en el movimiento de las especies. A mayor número de barreras, la similitud decrece más abruptamente que en un sitio topográficamente abierto y homogéneo (Nekola y White, 1999; Soininen et al., 2007). El tercero depende de la capacidad de dispersión de las especies pues la relación similitud-distancia ocurre aun si el ambiente es totalmente homogéneo, debido a las diferencias en la vagilidad de las especies (teoría de la neutralidad). Estos mecanismos no son mutuamente excluyentes y el patrón de decaimiento de la similitud con la distancia existe, probablemente en la mayoría de las comunidades, controlado conjuntamente por procesos basados en el nicho, en la configuración espacial y en la neutralidad (Nekola y White, 1999; Soininen et al., 2007).

En un trabajo reciente, Martiny et al. (2011) analizan el decaimiento de la similitud con la distancia, retoman las ideas generadas por Preston (1960) y analizan la relación entre el decaimiento con la distancia y la escala geográfica en bacterias de los sedimentos de marismas. Contrario a lo esperado, la distancia geográfica tuvo un fuerte efecto sobre la similitud de la comunidad dentro de las marismas (escala local), pero no entre marismas de una costa (escala regional), ni entre continentes. Como en los macroorganismos, los causantes de la diversidad $\beta$ de bacterias de las marismas depende de la escala espacial; sin embargo, en

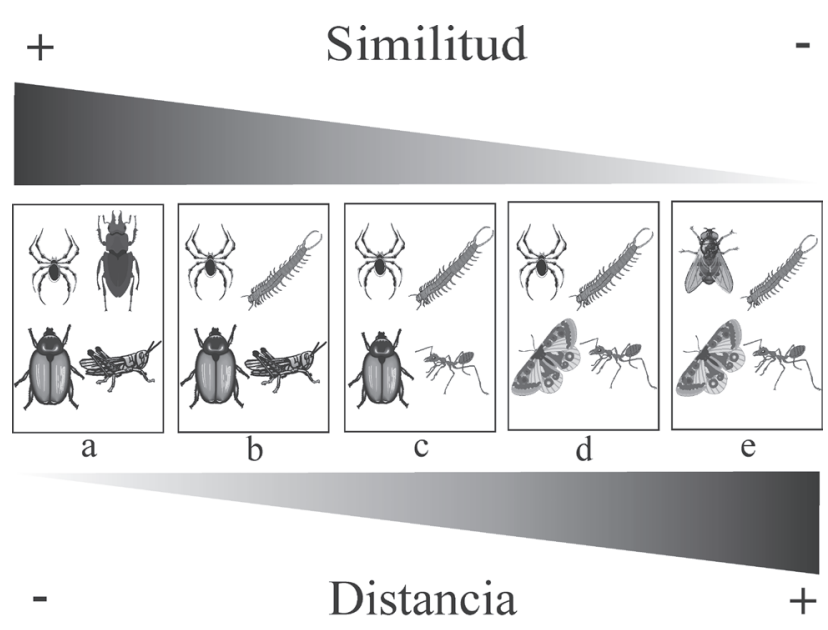

Figura 2. Patrón de decaimiento de la similitud entre comunidades conforme aumenta la distancia. Comunidades hipotéticas ( $a, b, c, d, e)$ ordenadas a través de gradiente espacial. La similitud en la composición de especies disminuye conforme aumenta la distancia entre las comunidades. 
bacterias no se presenta diversificación evolutiva de taxa a escala continental (Martiny et al., 2011).

$L a$ diversidad $\beta$ y el recambio de especies. No fue sino hasta el año 2001 cuando Vellend proporcionó evidencias estadísticas de que la diversidad $\beta$ per se y el recambio de especies son fenómenos diferentes (aunque evidentemente relacionados) que se deben utilizar y calcular de forma diferente. Vellend (2001) argumenta que el recambio debe conceptualizarse como la tasa o magnitud de cambio en la composición de especies a lo largo de gradientes ambientales o espaciales predefinidos, lo cual implica que existe algún tipo de estructura en el paisaje que modela la variación en la composición de especies entre los sitios de muestreo. De acuerdo con Vellend (2001), cuando el cambio en la composición de especies a lo largo de gradientes ambientales o espaciales es el principal interés, el uso de medidas como el índice de $\beta$ de Whittaker (1960), y medidas derivadas de este índice, son inapropiados, ya que la forma más adecuada para evaluar el recambio de especies es a través de relaciones de distancia y similitud que pueden calcularse entre pares de sitios de acuerdo con su disposición en el gradiente, lo cual los hace más confiables para detectar diferencias graduales en el recambio de especies (Vellend, 2001).

En trabajos recientes (Moreno y Rodríguez, 2010; Tuomisto, 2010a, 2010b, 2011; Anderson et al., 2011) se reconoce el recambio de especies como un fenómeno biológico particular; también se reconoce que el recambio de especies es uno de los fenómenos que se han albergado bajo el término sombrilla "diversidad $\beta$ " (Fig. 3). Para algunos, el recambio de especies es el cambio en la composición y estructura de las comunidades de una unidad de muestreo a otra a lo largo de un gradiente espacial, temporal o ambiental (Vellend, 2001; Moreno y Rodríguez, 2010; Anderson et al., 2011). Para Tuomisto (2010a, 2011) el recambio de especies incluye a todo un subgrupo de medidas de disimilitud o diferenciación en la composición de especies que pueden expresarse en función de la partición aditiva de la diversidad gamma (véase en Etapa 3, el apartado $\beta$ como un componente de la diversidad gamma y el concepto de $\beta$ "verdadera").

Otros fenómenos distintos al recambio de especies han permanecido bajo los términos " $\beta$ " (Vellend, 2001), " $\beta$ verdadera" (Tuomisto, 2010a, 2010b), "variación" (Anderson et al., 2011) y "diferenciación" de ensambles de especies (Jost et al., 2011). En estos fenómenos se incluye la diferenciación en la composición de especies cuando no hay un orden direccional en las muestras, por ejemplo cuando se analiza la diversidad $\beta$ entre los tipos de vegetación de un paisaje. La gran diferencia radica en que el recambio de especies implica una direccionalidad en la secuencia de las muestras, a raíz de un gradiente. Desde otro punto de vista, el recambio de especies se ha considerado como un componente, que junto con el anidamiento, dan lugar a la “diversidad $\beta$ ” (Baselga, 2010a), como se explica más adelante en el apartado $\beta=$ recambio + anidamiento.

Etapa 2. Proliferación de medidas y expansión del concepto. Después de las aportaciones iniciales de Whittaker (1956 y 1960) y MacArthur (1965), se fueron desarrollaron distintas aproximaciones para analizar el cambio en la composición de especies de las comunidades. En términos generales, esta etapa es donde se proponen diversas medidas de $\beta$ y donde se evidencia lo confuso del concepto. A continuación se mencionan algunas de las que a nuestro parecer incluyen gran parte de la vasta gama de valiosos trabajos realizados sobre la diversidad $\beta$ o fenómenos relacionados con ella.
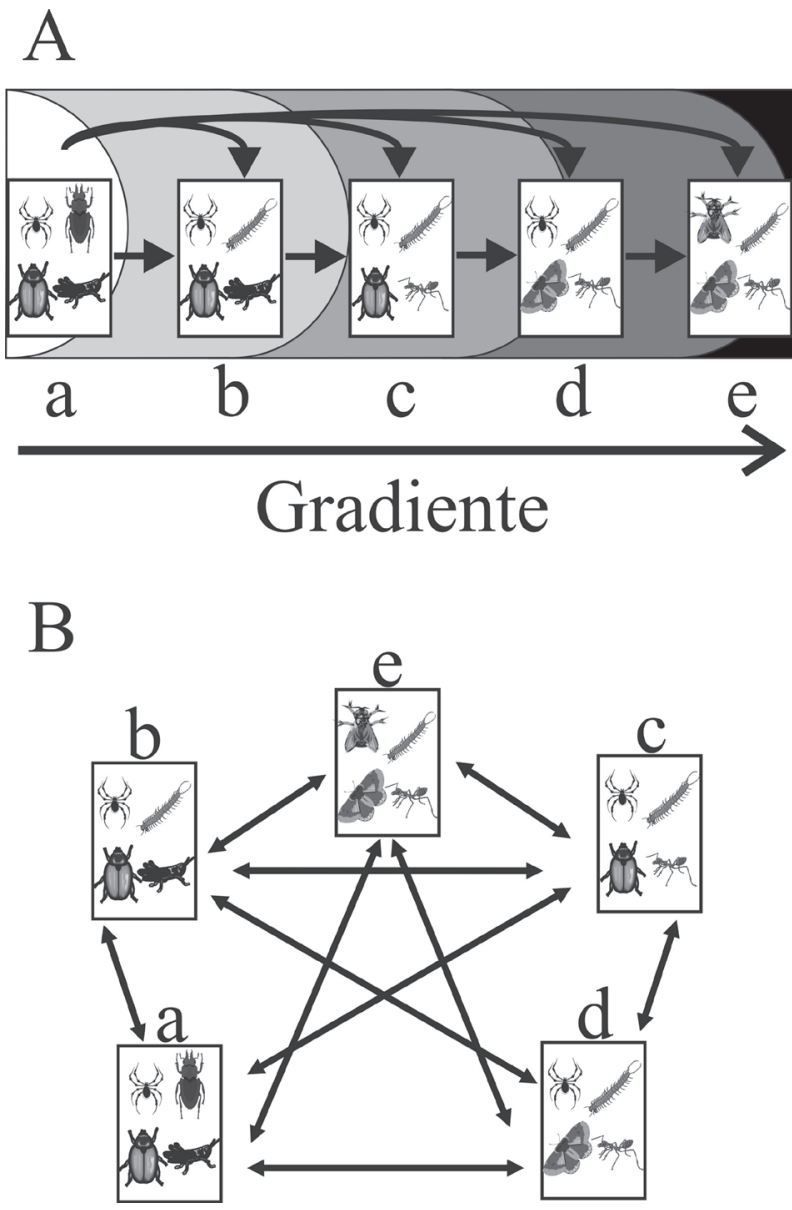

Figura 3. Fenómenos biológicos que han sido citados como diversidad beta. A, recambio de especies, cambio en la composición de especies entre comunidades ( $\mathrm{a}, \mathrm{b}, \mathrm{c}, \mathrm{d}, \mathrm{e})$, siguiendo la dirección de un gradiente espacial, temporal o ambiental; $\mathrm{B}$, diferenciación o variación, mide el cambio en la composición de especies entre comunidades que no están inmersas en un gradiente, y por lo tanto no hay direccionalidad en el orden del muestreo. 
Clasificación de las distintas variantes de la diversidad $\beta$. El primer intento de comparar diferentes medidas de diversidad $\beta$ fue el trabajo de Wilson y Shmida (1984), quienes analizan 6 índices de diversidad $\beta$ : el índice de Whittaker, el índice de Cody, 3 índices propuestos por Routledge y 1 propuesto por ellos mismos. Los criterios de evaluación del desempeño de estos índices fueron 4: 1) que el resultado del índice esté acorde con la noción intuitiva de recambio en la comunidad; 2) que posea la propiedad de aditividad; 3) que tenga independencia del valor de diversidad $\alpha$ y 4) que sea independiente del tamaño de muestra. Los resultados mostraron que el índice de Whittaker y el propuesto por ellos cumplieron con los 4 criterios de buen desempeño. Este trabajo lo retomó Magurran (1988) para sintetizar las diferencias entre los índices de diversidad $\beta$ existentes y sus propiedades.

Koleff et al. (2003a) realizaron una de las revisiones más completas de las medidas de diversidad $\beta$ utilizadas para datos de presencia-ausencia entre pares de sitios. Estos autores analizan 24 índices de diversidad $\beta$ de acuerdo con su respuesta en la variación de los porcentajes de 3 componentes básicos: 1) porcentaje de las especies compartidas por un par de sitios; 2) porcentaje de especies presentes exclusivamente en el sitio vecino y 3) porcentaje de especies presentes sólo en el sitio focal. Como resultado de este análisis, Koleff et al. (2003a) proporcionan una clasificación de índices en 4 grupos: 1) medidas de continuidad y pérdida; 2) medidas de gradientes de riqueza de especies; 3) medidas de continuidad y 4) medidas de ganancia y pérdida (medidas de recambio, que se enfocan en identificar diferencias en la composición más que diferencias en la riqueza de especies). Los autores señalan que el índice de diversidad $\beta$ más utilizado es el de Whittaker. Además, mencionan que la mayoría de los estudios sólo utilizan 1 índice de diversidad y que las medidas de diversidad $\beta$ menos utilizadas son las de ganancia y pérdida (recambio de especies). Los 4 grupos distinguidos en este trabajo para 24 índices mostraron la redundancia de los mismos, lo que evidenció la confusión en el uso de las medidas, que la conceptualización de la diversidad $\beta$ había sido insuficiente y que era necesario un trabajo más exhaustivo para evitar la proliferación de medidas similares.

Relaciones especie-área. Si bien la relación especies-área no surgió originalmente vinculada al concepto de diversi$\operatorname{dad} \beta$, la pendiente de dicha relación $(z)$, ha sido relacionada con la diferenciación en la composición de especies (Westoby, 1993; Rosenzweig, 1995; Crawley y Harral, 2001; Arita y Rodríguez, 2002; Rodríguez y Arita, 2004). Un valor alto de $z$ corresponde a una rápida acumulación de especies conforme se incrementa el área, indicando tasas elevadas de recambio de especies entre sitios que componen una región más grande (Arita y Rodríguez, 2002).
Además, se ha registrado una relación directa entre la pendiente de las relaciones especies-área y los patrones del tamaño de distribución de las especies. El valor de la pendiente no cambia en intervalos pequeños, pero sí lo hace cuando los intervalos son grandes, lo que implica que si la escala varía, también lo hacen los patrones de distribución de las especies y el recambio de las mismas. Esta forma de cuantificar la diversidad $\beta$ o recambio de especies ha sido utilizada por varios autores con diferentes grupos biológicos y distintos propósitos, aunque en la mayoría de los casos se ha empleado para evaluar el cambio en la composición de especies entre comunidades a través de un gradiente (Arita y Rodríguez, 2002; Qian y Ricklefs, 2007). Sin embargo, otros autores critican la validez de dicha pendiente como medida de diversidad $\beta$ (Connor y McCoy, 1979; Scheiner et al., 2011). Por supuesto, esta aproximación tiene un vasto potencial de aplicación en macroecología y biogeografía, por lo que merece aún un análisis profundo.

Anidamiento. Otra de las aproximaciones utilizadas para analizar el cambio en la composición de las comunidades es el anidamiento. Los análisis de conjuntos anidados fueron propuestos para describir patrones de composición de especies entre biotas continentales y entre hábitats aislados, como islas o paisajes fragmentados y el anidamiento fue atribuido a tasas diferenciales de extinción y colonización. Sin embargo, diversos fenómenos determinísticos o estocásticos pueden también llevar a la formación de un sistema anidado (Wright y Reeves, 1992; Ulrich et al., 2009). Se ha propuesto que el anidamiento puede ser resultado de factores como el muestreo pasivo, el anidamiento del hábitat, el aislamiento espacial y el área (Wright et al., 1998). El muestreo pasivo ocurre cuando los parches de diversos tamaños presentan una tendencia a ocuparse por las especies más abundantes o comunes, ya que si la probabilidad de que una especie colonice un sitio es igual a su abundancia regional, entonces las especies más abundantes tendrán una mayor probabilidad de colonizar muchos más parches de los que ocuparían especies con densidades bajas. Otro factor es el anidamiento del hábitat, causado principalmente por la fragmentación del mismo, en este caso las especies se encuentran anidadas porque sus hábitats también lo están. En el caso del aislamiento espacial, entre mayor distancia haya entre la comunidad fuente y la receptora de especies, el anidamiento será menor, puesto que a mayor aislamiento, mayor dificultad para que las especies superen esta barrera. Finalmente, el área también influye sobre el anidamiento ya que, en un conjunto de parches, los que presenten áreas mayores tendrán mayor número de especies y conforme va disminuyendo el área de los mismos también lo hace la riqueza (Wright et al., 1998). 
En términos generales, existen 3 restricciones principales que limitan el anidamiento, todas ellas relacionadas con variación en la homogeneidad, ya que cualquier causa que introduzca heterogeneidad en el sistema minimizará en algún grado el anidamiento (Wright y Reeves, 1992). La primera es la heterogeneidad en la distribución de las especies, que puede deberse a endemismos y a variación geográfica en las fuentes de especies. La segunda es la historia, pues el ensamblaje de las comunidades puede estar influenciado por eventos del pasado: si los sitios tienen diferentes historias biogeográficas o han presentado diferentes procesos de desarrollo de la comunidad, el resultado serán comunidades de especies heterogéneas. Y la tercera es la heterogeneidad espacial en el medio, que puede causar variación geográfica en la distribución de especies. También pueden ser importantes el estado o condición actual de los parámetros poblacionales de las especies y la calidad de los hábitats (Wright y Reeves, 1992).

Etapa 3. Hacia una síntesis conceptual de beta y fenómenos relacionados. Los trabajos mencionados con anterioridad proporcionan evidencia de la proliferación de conceptos y métodos para medir la diversidad $\beta$. A continuación se exponen las principales novedades conceptuales y metodológicas surgidas recientemente, tales como el análisis de patrones espaciales de la diversidad $\beta$ y de los factores que pueden determinar sus causas, la predicción de especies compartidas entre 2 comunidades de Chao et al. (2005), la partición de la diversidad $\beta$ en sus componentes de recambio y anidamiento de Baselga (2010a), así como la partición aditiva y multiplicativa de la diversidad $\gamma \mathrm{y}$ el concepto de la diversidad verdadera de Jost (2006, 2007). Además, se presentan las revisiones conceptuales más importantes propuestas por Jurasinski et al. (2009), Tuomisto (2010 a, b y c) y Anderson et al. (2011).

Explicación de los factores que originan e influyen en la diversidad $\beta$. Además de la búsqueda de conceptos y medidas uniformes del cambio en la composición de especies entre comunidades, se han propuesto métodos para conocer los fenómenos que originan este cambio. De acuerdo con Legendre et al. (2005), las principales hipótesis con las que actualmente contamos para explicar las causas de la diversidad $\beta$ son: 1) la composición de especies es uniforme sobre áreas grandes, por lo que la diversidad $\beta$ es nula; 2) la composición de especies fluctúa aleatoriamente en una forma autocorrelacionada dada la capacidad de dispersión de las especies (dispersión histórica) y 3) la distribución de las especies está relacionada con las condiciones ambientales (control ambiental). Estas hipótesis pueden ser probadas por medio de la partición de la variación de la composición en las comunidades con respecto a funciones de variables ambientales y espaciales, a través de 2 enfoques basados en estadística multivariada. El pri- mero es el enfoque de Mantel, con el cual se analiza la correlación (prueba de Mantel) entre la matriz de similitud en la composición de especies entre muestras, y la matriz de similitud en variables ambientales entre muestras. El segundo enfoque consiste en utilizar la suma de cuadrados de una base de datos de la composición de una comunidad, la cual se particiona correctamente por ordenación canónica (Legendre et al., 2005). Este es el enfoque llamado de "datos crudos" (raw data approach) porque la información inicial del análisis es una tabla con los datos de abundancia por especie de cada sitio de muestreo, y otra tabla con medidas de variables ambientales en los mismos sitios de estudio. Este segundo enfoque permitió a Legendre et al. (2005) generar un concepto de $\beta$ diferente, definiéndola como "la varianza en la composición de especies entre sitios en una región geográfica". Además, Legendre et al. (2005) proponen un marco conceptual para entender las causas que originan la diversidad $\beta$ en 3 niveles de abstracción: 1) variación en la identidad de las especies en el nivel de comunidades (diversidad $\alpha$ ); 2) variación de la composición de las comunidades entre sitios (diversidad $\beta)$ y 3) variación en la diversidad $\beta$ entre grupos de sitios. Dichos autores concluyen que para el segundo nivel de abstracción, la partición canónica es el procedimiento adecuado para dividir la variación de la composición de una comunidad entre componentes espaciales y ambientales, y para probar hipótesis sobre el origen y mantenimiento de la variación entre sitios, mientras que el enfoque de Mantel es apropiado para hipótesis relacionadas con el tercer nivel de abstracción (Legendre et al., 2005). Tuomisto y Roukolainen (2006) criticaron la propuesta anterior, difiriendo en el uso de términos básicos, como analizar y explicar, ya que consideran que estos términos llevan implícitos distintos razonamientos y la forma en que fueron utilizados por Legendre et al. (2005) confunde y provoca que se generen percepciones equivocadas de la forma en que se analiza el cambio en la composición de especies de una base de datos. Tuomisto y Roukolainen (2006) también difieren en los niveles de abstracción propuestos por Legendre et al. (2005); argumentan que el primer nivel no debe ser igual a la diversidad $\alpha$ si no que se debe hablar de composición, ya que la riqueza puede ser la misma pero no la composición.

El artículo de Tuomisto y Roukolainen (2006) generó un foro de discusión en la revista Ecology (Laliberté, 2008; Legendre et al., 2008; Pélissier et al., 2008; Tuomisto y Ruokolainen, 2008). Las conclusiones del mismo apoyaron la propuesta original de Legendre et al. (2005), argumentando que el uso de distintos términos básicos no alteraba de manera puntual los resultados de su propuesta de análisis, y que la modificación de Tuomisto y Roukolainen (2006) a los niveles de abstracción no responde a las preguntas expresadas por Legendre et al. (2005) sobre 
la validez matemática de la partición de la variación sobre matrices de distancia, debido a que se aplicó una definición inadecuada del concepto de autocorrelación espacial (Laliberté, 2008). En términos generales, las propuestas de Tuomisto y Ruokolainen $(2006,2008)$ no demostraron ser mejores que la propuesta original de Legendre et al. (2005); por lo tanto, la propuesta de estos autores permanece vigente como una importante aportación a la forma de analizar la diversidad $\beta$ y los factores que la generan.

Predicción de la diversidad $\beta$ entre comunidades con esfuerzo de muestreo insuficiente. Una opción que consideramos interesante para complementar los análisis de la diversidad $\beta$ son los estimadores del número de especies compartidas entre 2 comunidades propuestos por Chao et al. (2005). Dichos autores mencionan que cuando se evalúa la diversidad $\beta$ entre comunidades no se suele considerar que, si el esfuerzo de muestreo es insuficiente, pueden existir especies que aún no han sido registradas en los inventarios, y estas especies faltantes podrían ser compartidas entre las comunidades. En términos generales, cuando el esfuerzo es insuficiente para registrar inventarios completos, la diversidad $\beta$ tiende a sobreestimarse. Por ello, la propuesta tiene gran relevancia ya que permite estimar cuántas especies faltan por registrar en cada comunidad, y cuántas de éstas podrían compartirse entre comunidades, derivando así estimadores del cambio en la composición de especies entre 2 comunidades. Estos estimadores se encuentran incluidos en el programa SPADE (Chao y Shen, 2010) y en el programa EstimateS (Colwell, 2009).

$\beta=$ recambio + anidamiento. El análisis de conjuntos anidados se ha utilizado como herramienta ecológica para describir patrones de presencia de especies y de las causas que los provocan. En un sistema anidado (Fig. 4), la composición de especies en ensambles pequeños es una submuestra de la composición de especies de ensambles grandes (Ulrich et al., 2009). Por ejemplo, la fragmentación del hábitat genera patrones anidados porque los paisajes quedan caracterizados por parches que difieren en tamaño y en su grado de aislamiento (Martínez-Morales, 2005). De acuerdo con algunos autores, el anidamiento dentro de paisajes fragmentados lo causan principalmente secuencias ordenadas de extinción (Ulrich et al., 2009). Sin embargo, todas las explicaciones para los subconjuntos anidados dentro de matrices geográficas pueden percibirse como variaciones ordenadas de extinción y colonización a lo largo de gradientes ambientales o biológicos de los parches bajo estudio.

Una de las aportaciones más recientes al análisis de la diversidad $\beta$ la realizó Baselga (2010a), al proporcionar evidencia de que la diversidad $\beta$ puede entenderse como el resultado de 2 fenómenos: la diferenciación en la composición de especies (fenómeno al que Baselga llama turnover o recambio) y el anidamiento entre comunidades con diferente número de especies. Contrario al anidamiento, el recambio implica la sustitución de unas especies por otras como consecuencia del arreglo espacial o de restricciones espaciales y/o históricas. Baselga (2010a) mide la diversidad $\beta$ total para un conjunto de comunidades con el índice de similitud de Sorensen ( $\beta$ sor), con el cual se puede describir la diferenciación espacial y las diferencias en riqueza de especies entre las comunidades (Koleff et al., 2003a). El principal aporte de Baselga es que logra dividir esta diversidad $\beta$ total en 2 componentes independientes: 1) la diferenciación en la composición de especies, medida con el índice de Simpson ( $\beta \operatorname{sim})$, que describe la variación sin la influencia de gradientes de riqueza y 2) el anidamiento ( $\beta$ nes). Baselga (2010a) propone el índice $\beta$ nes para el componente de anidamiento con el siguiente razonamiento: cuando 2 comunidades presentan la misma riqueza de especies, no existe anidamiento entre ellas, por lo que

\section{A Sistema anidado}

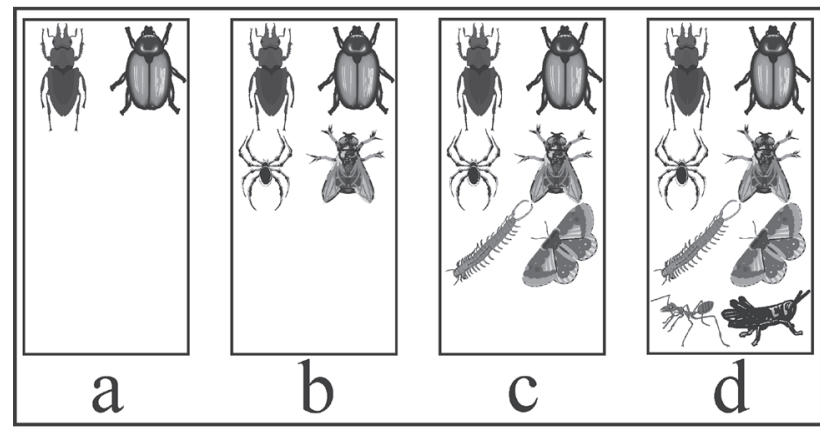

\section{B Sistema no anidado}

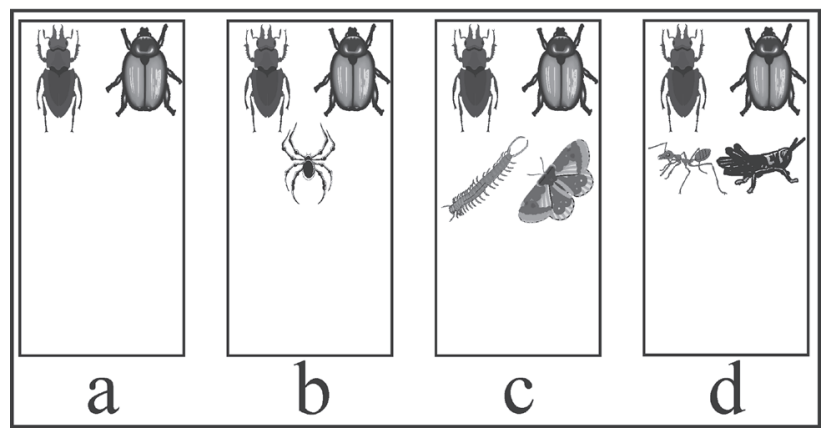

Figura 4. Comunidades (a, b, c, d) con distinto grado de anidamiento en su composición de especies. A, sistema anidado; la composición en comunidades con pocas especies es una submuestra de la composición en comunidades más ricas en especies; $\mathrm{B}$, sistema no anidado; las comunidades ricas en especies no incluyen todas las presentes en las comunidades con menor riqueza. 
la diversidad $\beta$ deberá ser originada por diferencias en la composición de especies. En estas circunstancias, el resultado de $\beta$ sor y $\beta$ sim es el mismo. Sin embargo, cuando las comunidades presentan un número diferente de especies, el resultado de ambos índices ya no es el mismo; por lo tanto, no toda la diversidad $\beta$ será originada por diferencias en composición de especies y esto significa que el factor de anidamiento se encuentra presente. Baselga (2010a) aplica una partición aditiva de la diversidad $\beta$, tanto para pares de sitios como para múltiples sitios.

Leprieur et al. (2011) utilizan la aproximación de Baselga (2010a) para estudiar los patrones de diversidad $\beta$ de peces de agua dulce a escala global. Detectan que la contribución relativa de la diferenciación en la composición de especies y del anidamiento depende de la ubicación geográfica, y encuentran patrones que reflejan la influencia de cambios climáticos durante el Cuaternario. En dicho trabajo, la partición aditiva de la diversidad $\beta$ en sus componentes (diferenciación y anidamiento) es esencial para revelar el papel de los factores históricos y ecológicos en los patrones actuales de diversidad $\beta$.

La aplicación de esta propuesta resulta particularmente importante en el manejo y conservación regional, ya que además de saber cuál sitio presenta mayor diversidad $\alpha$ dentro del paisaje, es importante conocer si la diversidad $\beta$ entre sitios es producto del anidamiento, de la diferenciación en la composición de especies, o de ambos fenómenos. La diferenciación en la composición implica la presencia de barreras o patrones de diferenciación selectiva entre las biotas, mientras que el anidamiento no. Para las estrategias de manejo, la presencia de una elevada diferenciación requiere la selección de múltiples sitios para la conservación, mientras que el anidamiento podría indicar que un número reducido es adecuado (Baselga, 2010a).

Sin embargo, Almeida-Neto et al. (2012) critican el método propuesto por Baselga y argumentan que en realidad $\beta$ nes no mide el anidamiento y que este índice es sensible al tamaño de la base de datos y a la forma del llenado de la misma, por lo que el índice puede cambiar su valor cuando el anidamiento no lo hace. De acuerdo con Almeida-Neto et al. (2012), la aportación de Baselga es útil para determinar cómo las diferencias en la riqueza de especies que no son originadas por el recambio de especies contribuyen con la diversidad $\beta$ a lo largo de gradientes espaciales, temporales o ambientales; sin embargo, no lo es para cuantificar el anidamiento, ya que éste es un caso especial de disimilitud en la composición de especies debido a una pérdida o ganancia ordenada de especies.

$\beta$ como un componente de la diversidad $\gamma$ el concepto de $\beta$ "verdadera". Whittaker (1960) planteó el siguiente razonamiento: si conocemos el promedio de la diversidad dentro de un conjunto de comunidades o muestras (diver- sidad $\alpha$ ), se puede obtener la diversidad total representada por la totalidad de las muestras (diversidad $\gamma$ ) como el resultado de la multiplicación de la diversidad $\alpha$ por la diversidad $\beta$. Este razonamiento $(\gamma=\alpha \times \beta)$ se conoce como la partición multiplicativa de la diversidad $\gamma$ en sus componentes $\alpha$ y $\beta$. La medida de diversidad $\beta$ derivada de este razonamiento $(\beta=\gamma / \alpha)$ ha sido muy utilizada en ecología y biogeografía y corresponde a cuántas veces es más rica en especies una base de datos completa (diversidad $\gamma$ o regional) que la riqueza que las unidades tienen en promedio (diversidad $\alpha$ promedio o local), por lo que es una medida de diferenciación relativa entre las diversidades $\alpha$ y $\gamma$ (Tuomisto, 2010a).

Alternativamente, Lande (1996) propuso que la diversidad total de especies en una región $(\gamma)$ puede ser dividida en componentes aditivos, de tal forma que la diversidad $\gamma$ sea la suma de las diversidades $\alpha$ y $\beta$ expresadas en las mismas unidades (partición aditiva de la diversidad $\gamma$ en sus componentes $\alpha$ y $\beta$ : $\gamma=\alpha+\beta$; de la cual se deriva que $\beta=\gamma-\alpha$ ). Este modelo aditivo se puede calcular con base en la riqueza de especies o en índices de diversidad, como el de Shannon o el de Gini-Simpson (Lande, 1996; Veech et al., 2002). Los impulsores de esta propuesta argumentan que las medidas tradicionales de $\beta$ (las de $\gamma / \alpha$, y aquellas basadas en el recambio o en la ganancia y pérdida de especies) proveen una estimación de $\beta$ que no es comparable con $\alpha$ debido a que las unidades de ambas no son iguales: para $\alpha$ el número de especies es la unidad, pero $\beta$ es adimensional (Veech et al., 2002). Además, muchas veces la diversidad $\beta$ se deriva de comparaciones entre pares de muestras, mientras que la diversidad $\alpha$ promedio se calcula simultáneamente a partir de todas las muestras. Por su parte, la diversidad $\beta$ medida por medio de la partición aditiva se expresa en las mismas unidades que las diversidades $\alpha$ y $\gamma$, por lo que puede compararse con ellas directamente (Veech et al., 2002). El valor de diversidad $\beta$ obtenido mediante la partición aditiva de $\gamma$ se refiere a la cantidad absoluta o total por la cual la diversidad $\gamma$ o regional excede la diversidad promedio de las unidades de muestreo (Tuomisto, 2010a).

De acuerdo con Jost (2007), la partición aditiva de la diversidad presenta varias desventajas. Primero, cuando la diversidad se mide con medidas clásicas, como el índice de entropía de Shannon y el de Gini-Simpson y sus resultados con la partición aditiva en algunos casos son inadecuados. Segundo, para medidas de diversidad divididas por partición aditiva, la similitud entre sus componentes $\alpha$ y $\gamma$ tiende a alcanzar la unidad (similitud total) cuando la diversidad $\alpha$ es alta, independientemente de la similitud entre los grupos. Tercero, cuando hay muchas comunidades, cada una dominada por una sola especie, la similitud aditiva resultante del índice de Gini-Simpson tiende a acercarse 
a cero indicando una muy alta o completa diferenciación. Para evitar este tipo de problemas, Jost $(2006,2007)$ deriva las expresiones matemáticas correctas que cumplen con 5 supuestos básicos: 1) la diversidad $\beta$ puede variar independientemente del valor de $\alpha$; 2) las diversidades $\alpha, \beta$ у $\gamma$ pueden ser directamente comparables; 3) la diversidad $\alpha$ es la diversidad promedio obtenida de todas las comunidades del paisaje; 4) la diversidad $\gamma$ debe ser determinada completamente por las diversidades $\alpha$ y $\beta$, y 5) la diversidad $\alpha$ nunca podrá ser mayor que la diversidad $\gamma$. Para que una medida de diversidad cuente con estas propiedades, Jost $(2006,2007)$ propuso un marco matemático que permite la derivación de una definición general de diversidad $\beta$ con números efectivos (también llamados números equivalentes). Los números efectivos son el número de elementos igualmente comunes, necesarios para producir un valor dado de diversidad. Siguiendo esta aproximación, la diversidad $\beta$ "verdadera" (true beta diversity, de acuerdo con Jost, 2007) se mide como el número efectivo de comunidades o unidades de muestreo diferentes en la región, que corresponde al número de comunidades "virtuales" que podrían encontrarse en la región, cuando todas ellas fueran distintas en su composición de especies y mantuvieran la misma diversidad $\alpha$ promedio observada entre las comunidades de la región. Estas mismas unidades de medición fueron nombradas posteriormente "unidades de composición" (compositional units) por Tuomisto (2010a). En resumen, la diversidad $\beta$ verdadera se obtiene cuando el número efectivo total de especies en un conjunto de datos (diversidad $\gamma$ verdadera) se divide entre el número efectivo de especies promedio de las muestras (diversidad $\alpha$ verdadera). Bajo este esquema, la diversidad $\beta$ verdadera tiene una interpretación uniforme, independientemente de cuál sea el índice de diversidad usado. La diversidad $\beta$ mínima posible es una comunidad efectiva, cuando todas las comunidades son exactamente iguales, mientras que el número máximo posible es igual al número de comunidades totales $N$, cuando todas ellas son completamente diferentes en su composición de especies (Jost, 2006, 2007).

A diferencia de los índices de diversidad comunes, donde en ocasiones sus resultados no coinciden con patrones de diversidad intuitivamente esperados, los números efectivos sí dan resultados coherentes y cumplen con una serie de axiomas indispensables para una evaluación matemática robusta. Por ejemplo, las medidas de diversidad verdadera cumplen con el principio de replicación, según el cual, si tenemos $N$ comunidades con igual riqueza de especies y con la máxima equidad en la distribución de sus abundancias, y si ninguna especie se comparte entre comunidades, entonces la diversidad del conjunto de estas comunidades será exactamente igual a $N$ veces la diversidad de una sola comunidad (Jost, 2007; Jost et al., 2010).
Recientemente, Baselga (2010b) y Jost (2010) ponen a prueba la independencia matemática de las particiones aditiva y multiplicativa de la diversidad $\gamma$ en sus componentes $\alpha$ y $\beta$. Ambos autores realizan su análisis con diferentes métodos y llegan a la misma conclusión: la única partición de la diversidad $\gamma$ que produce un componente $\beta$ totalmente independiente de la diversidad $\alpha$ es la partición multiplicativa. A pesar de que Veech y Crist (2010) y Anderson et al. (2011) argumentan que aún existe debate en cuanto a si la partición debe ser aditiva o multiplicativa, ya que consideran que ambas pueden ser útiles a pesar de sus limitaciones, distintos autores concuerdan en que la única forma de tener independencia entre los componentes $\alpha$ y $\beta$ es por medio de la partición multiplicativa (Jost, 2006, 2007, 2010; Jost et al., 2010; Baselga, 2010b; Tuomisto, 2010a, 2010b, 2010c). Esta independencia es necesaria para analizar los procesos ecológicos y biogeográficos que originan y mantienen los componentes $\alpha$ y $\beta$ (Wilson y Shmida, 1984; Baselga, 2010b).

Otra ventaja importante de la derivación de la diversidad $\beta$ verdadera es que está íntimamente conectada con medidas de diferenciación en la composición de especies y de similitud entre comunidades (Jost, 2007; Jost et al., 2011). A través de distintas transformaciones, la diversidad $\beta$ verdadera se relaciona con índices de similitud de uso común, como los de Jaccard, Sorensen y Morisita-Horn, por lo que es posible llegar a un marco unificado de análisis bajo el esquema de la diversidad $\beta$ verdadera. Sin embargo, otros índices habituales como el de Bray-Curtis no se relacionan analíticamente con la diversidad $\beta$, por lo cual las conclusiones basadas en estos índices pueden resultar contrarias a las obtenidas con medidas de diversidad $\beta$ verdadera (Jost, 2007; Jost et al., 2011).

Revisiones recientes. En los últimos años aparecieron varios trabajos con aportaciones novedosas que han contribuido en la búsqueda de la consolidación y unificación de criterios de la diversidad $\beta$ y de los fenómenos que le han sido asociados. A continuación se mencionan las aportaciones más importantes. .

Jurasinski et al. (2009) realizaron una revisión de conceptos confundidos con el término diversidad $\beta$, para lo cual analizan 73 publicaciones; mencionan que hay 2 grupos de medidas para la diversidad $\beta$ : un grupo que incluye medidas que examinan la variación en la riqueza de especies (expresados como número de especies o por índices de riqueza a través de escalas), incluyendo la diversidad $\beta$, derivada tanto de la partición multiplicativa como de la partición aditiva. El otro grupo incluye coeficientes que analizan la variación en la composición de especies entre muestras (tomando en cuenta la distinción de las especies y/o su abundancia), donde se incluye la semejanza expresada por coeficientes de similitud/disimilitud. 
Moreno y Rodríguez (2010) critican parcialmente esta clasificación argumentando que el recambio de especies no pudo ser identificado adecuadamente por Jurasinski et al. (2009) debido a algunas omisiones importantes en la revisión de literatura realizada por estos autores, que resultó ser incompleta. Por su parte, Toumisto (2010c) hace un comentario sobre la propuesta de Moreno y Rodríguez (2010) coincidiendo en que el concepto de diversidad $\beta$ se debe esclarecer y el recambio de especies diferenciarse de la diversidad $\beta$ per se. En su comentario, Toumisto (2010c) hace una síntesis y explica con analogías el concepto de diversidad $\beta$ verdadera, ampliamente desarrollado en sus trabajos previos (Tuomisto, 2010a, 2010b).

En la compilación de Tuomisto (2010a, 2010b), constituida por 2 artículos seriados, se analizan desde el punto de vista teórico-matemático los conceptos y métodos para medir los diferentes fenómenos que se han albergado bajo el término "diversidad $\beta$ ", retomando las aportaciones más importantes a la fecha. Uno de los puntos centrales de estos trabajos es la separación de las diferentes medidas y métodos para cuantificar la diversidad $\beta$ verdadera (véase sección: $\beta$ como un componente de la diversidad gamma $y$ el concepto de $\beta$ "verdadera"), de las medidas que la autora denomina como "otros fenómenos relacionados con $\beta$ ”. En la segunda parte de su trabajo, Tuomisto (2010b) analiza matemáticamente 5 formas diferentes de medir la diversidad $\beta$, para lo cual forma 2 grandes grupos: $a$ ) las que miden la heterogeneidad en la composición de la base de datos y b) las que miden el recambio efectivo de especies. En el primer caso se incluye la diversidad $\beta$ verdadera y las tasas de la diversidad regional a la local; la primera mide el número de unidades de composición (comunidades efectivas) y la segunda, cuántas veces es más rica la región en especies efectivas que una comunidad promedio (Tuomisto, 2010b). El recambio efectivo de especies (cambio en la composición efectiva de la comunidad) incluye a su vez otros 3 fenómenos: 1) recambio absoluto de especies efectivas, que se refiere al número acumulado de especies efectivas que cambian entre todas las unidades composicionales de la base de datos; 2) el recambio de especies efectivas de Whittaker, que cuantifica el número de veces que la composición de especies efectivas cambia completamente a lo largo de todas las unidades composicionales y 3) recambio proporcional de especies efectivas, que mide qué proporción de la composición de especies efectivas de las base de datos completa cambia entre las unidades composicionales.

Otra clasificación de métodos relacionados con la diversidad $\beta$ es la presentada en el trabajo de Anderson et al. (2011), quienes también revisan los conceptos más importantes de la diversidad $\beta$, así como los diseños de muestreo necesarios para estudiarla, dependiendo de la pregunta a contestar y de los datos e información disponibles. Anderson et al. (2011) inician su trabajo separando claramente 2 conceptos albergados bajo el término "diversidad $\beta$ ", retomando la propuesta de Vellend (2001): recambio de especies (turnover) y variación (variation, a lo que Vellend, 2001, denomina diversidad $\beta$ ) (Fig. 3). Anderson et al. (2011) sugieren el uso de medidas clásicas de diversidad $\beta$, derivadas de la relación entre la diversidad $\alpha$ y $\gamma, \mathrm{y}$ también el uso de medidas multivariadas.

Una de las principales aportaciones de este trabajo es que, tanto para el concepto de recambio de especies como para el concepto de variación, proporciona esquemas conceptuales y de diseño de muestreo que permiten visualizar la pregunta de interés, la forma de abordarla y algunos métodos disponibles para analizarla. Anderson et al. (2011) analizan las implicaciones del uso de datos de presencia-ausencia (binarios) y el uso de datos de abundancia, y concluyen que los distintos tipos de datos son útiles para resaltar distintos fenómenos biológicos en la composición de las comunidades y pueden ser utilizados de acuerdo con la respuesta que se desee obtener. En sus conclusiones, Anderson et al. (2011) sugieren que el usuario utilice diferentes métodos para tratar de responder su pregunta, y de esta forma se podrán detectar cuáles son las mejores respuestas. Sin embargo, consideramos que esta sugerencia puede originar grandes confusiones y es necesario hacer hincapié en que la selección de los métodos de análisis debe basarse en una documentación profunda de sus implicaciones y supuestos, y que el uso de varios métodos puede confundir más que ayudar a su esclarecimiento. Conclusiones y perspectivas

Dada la amplia variedad de conceptos y fenómenos biológicos que han sido estudiados bajo el término "diversidad beta”, cada uno analizado con métodos particulares, resulta muy complicado intentar obtener generalizaciones sobre el tema. Las discusiones recientes (especialmente en la última década) acerca de los conceptos y métodos utilizados para cuantificar la diversidad $\beta$, han sido fructíferas, ya que han permitido que el concepto sea analizado a fondo, registrando componentes inmersos en él que no habían sido reflexionados con anterioridad. Estos avances han dejado atrás el calificativo de Cenicienta atribuido a la diversidad $\beta$ por Novotny y Weiblen (2005) y le han dado un papel protagónico en los estudios de ecología de comunidades y ecología geográfica, con un potencial importante de aplicación en biología de la conservación.

Nuevas ideas, y sobre todo nuevos métodos de análisis, seguirán generándose para fortalecer las bases teóricas de la diversidad $\beta$. Por ejemplo, todo lo que se ha presentado en este trabajo está basado en medidas neutrales de diversidad, que no consideran ningún tipo de diferenciación entre las especies de una comunidad. Aún es incipiente, pero ya 
hay algunos trabajos que ofrecen medidas de la diversidad no neutrales para incluir, por ejemplo, diferenciación entre las especies por su historia evolutiva (Chao et al., 2010; Vellend et al., 2011). La diversidad $\beta$ filogenética mide cómo varían las relaciones filogenéticas en el espacio o en el tiempo, de forma análoga a la manera en que la diversidad $\beta$ mide cómo cambia la composición de especies (Graham y Fine, 2008). Constituye una línea de investigación novedosa para entender cómo cambia la estructura evolutiva de las comunidades, y al utilizarla como marco conceptual para algunos tópicos en ecología y evolución, abre la oportunidad de avanzar en el entendimiento de los mecanismos que originan y mantienen los patrones de la biodiversidad (Graham y Fine, 2008). Por otro lado, la diversidad $\beta$ funcional mide la variabilidad en los caracteres funcionales de las especies entre distintos ensamblajes (Ricotta y Burrascano, 2008). Con esta filosofía de evaluación de las medidas de diversidad no neutrales continuarán desarrollándose distintas facetas de la diversidad $\beta$, como aproximaciones emergentes que brindarán insumos frescos para entender la diferenciación entre comunidades.

En este trabajo hemos compendiado las principales tendencias de la medida a lo largo de sus primeros 50 años. Por lo pronto, es una revisión actualizada del tema, incluyente de los conceptos y propuestas generales de estudio, pero excluyente de la explicación matemática de las medidas de análisis. La tarea pendiente es la revisión y aplicación correcta de los métodos disponibles, dependiendo de la pregunta, por parte de los usuarios que se interesen en el estudio práctico de la diversidad beta.

\section{Agradecimientos}

Las opiniones vertidas en este trabajo son el resultado de charlas con muchos colegas y amigos, a quienes estamos muy agradecidos por compartir sus ideas y resultados de investigación. Dos revisores anónimos aportaron ideas que mejoraron notablemente el contenido y la presentación del manuscrito. J. Calderón recibió una beca (374658/250634) del CONACYT para realizar sus estudios doctorales y este trabajo es parte de su tesis. La realización de este artículo fue posible gracias al apoyo del proyecto de ciencia básica SEP/CONACYT 84127.

\section{Literatura citada}

Almeida-Neto, R., D. M. B. Frensel y W. Ulrich. 2012. Rethinking the relationship between nestedness and beta diversity: a comment on Baselga (2010). Global Ecology and Biogeography 21:772-777.

Anderson, M. J., T. O. Crist, J. M. Chase, M. Vellend, B. D. Inouye, A. L. Freestone, N. J. Sanders, H. V. Cornell, L.
S. Comita, K. F. Davies, S. P. Harrison, N. J. B. Kraft, J. C. Stegen y N. G. Swenson. 2011. Navigating the multiple meanings of $\beta$ diversity: a roadmap for the practicing ecologist. Ecology Letters 14:19-28.

Arita, H. y P. Rodríguez. 2002. Geographic range, turnover rate and the scaling of species diversity. Ecography 25:541-550.

Baselga, A. 2010a. Partitioning the turnover and nestedness components of beta diversity. Global Ecology and Biogeography 19:134-143.

Baselga, A. 2010b. Multiplicative partition of true diversity yields independent alpha and beta components; additive partition does not. Ecology 91:1974-1981.

Chao, A., R. L. Chazdon, R. K. Colwell y T. J. Shen. 2005. Un nuevo método estadístico para la evaluación de la similitud en la composición de especies con datos de incidencia y abundancia. In Sobre diversidad biológica: el significado de las diversidades alfa, beta y gamma. m3m-Monografías Tercer Milenio, vol. 4, G. Halffter, J. Soberón, P. Koleff y A. Melic (eds.). Sociedad Entomológica Aragonesa (SEA)/ Comisión Nacional para el Conocimiento y Uso de la Biodiversidad/ Grupo Diversitas México y Consejo Nacional de Ciencia y Tecnología, Zaragoza. p. 85-96.

Chao, A. y T. J. Shen. 2010. Program SPADE (Species Prediction and Diversity Estimation). Program and user's guide. http:// chao.stat.nthu.edu.tw/softwareCE.html; última consulta: 18.V.2011.

Chao, A., Ch-H. Chiu y L. Jost. 2010. Phylogenetic diversity measures based on Hill numbers. Philosophical Transactions of the Royal Society B 365:3599-3609.

Cody, M. L. 1975. Towards a theory of continental species diversities: bird distribution over Mediterranean habitat gradients. In Ecology and Evolution of Communities, M. L. Cody y J. M. Diamond (eds.). Harvard University Press, Cambridge, Massachusetts. p. 214-257.

Colwell, R. K. 2009. EstimateS: Statistical Estimation of Species Richness and Shared Species from Samples, ver.8.2. User's guide and application. http://viceroy.eeb. uconn.edu/estimates; última consulta: 18.V.2011.

Connor, E. F. y E. D. McCoy. 1979. The statistics and biology of the species area-relationships. American Naturalist 113:791-833.

Crawley, M. J. y J. E. Harral. 2001. Scale dependence in plant biodiversity. Science 291:864-868.

Graham, C. H. y P. V. A. Fine. 2008. Phylogenetic beta diversity: linking ecological and evolutionary processes across space in time. Ecology Letters 11:1265-1277.

Jost, L. 2006. Entropy and diversity. 2006. Oikos 113:363-375.

Jost, L. 2007. Partitioning diversity into independent alpha and beta components. Ecology 88:2427-2439.

Jost, L. 2010. Independence of alpha and beta diversities. Ecology 91:1969-1974.

Jost, L., P. De Vries, T. Walla, H. Greeney, A. Chao y C. Ricotta. 
2010. Partitioning diversity for conservation analyses. Diversity and Distributions 16:65-76.

Jost, L., A. Chao y R. L. Chazdon. 2011. Compositional similarity and $\beta$ (beta) diversity. In Biological diversity: frontiers in measurement and assessment, A. Magurran y B. J. McGill (eds.). Oxford University Press, New York. p. 66-87.

Jurasinski, G., V. Retzer y C. Beierkuhnlein. 2009. Inventory, differentiation, and proportional diversity: a consistent terminology for quantifying species diversity. Oecologia 159:15-26.

Koleff, P., K. Gaston y J. Lennon. 2003a. Measuring beta diversity for presence-absence data. Journal of Animal Ecology 72:367-382.

Koleff, P., J. Lennon y K. Gaston. 2003b. Are there latitudinal gradients in species turnover? Global Ecology and Biogeography 12:483-488.

Laliberté, E. 2008. Analyzing or explaining beta diversity? Comment. Ecology 89:3232-3237.

Lande, R. 1996. Statistics and partitioning of species diversity and similarity among multiple communities. Oikos 76:5-13.

Legendre, P., D. Borcard y P. R. Peres-Neto. 2005. Analyzing beta diversity: partitioning the spatial variation of community composition data. Ecological Monographs 75:435-450.

Legendre, P., D. Borcard y P. R. Peres-Neto. 2008. Analyzing or explaining beta diversity? Comment. Ecology 89:32383244.

Leprieur, F., P. A. Tedesco, B. Hugueny, O. Beauchard, H. H. Durr, S. Brosse y T. Oberdorff. 2011. Partitioning global patterns of freshwater fish beta diversity reveals contrasting signatures of past climatic changes. Ecology Letters 14:325-334.

MacArthur, R. H. 1965. Patterns of species diversity. Biological Review 40:510-533.

Magurran, A. E. 1988. Ecological diversity and its measurement. Princeton University Press, New Jersey. 192 p.

Martínez-Morales, M. A. 2005. Nested species assemblages as a tool to detect sensitivity to forest fragmentation: the case of cloud forest birds. Oikos 108:634-642.

Martiny, J. B. H., J. A. Eisen, K. Penn, S. D. Allison y M. C. Horner-Devine. 2011. Drivers of bacterial $\beta$-diversity depend on spatial scale. Proceedings of the National Academy of Science of the United States of America 108:785-7854.

Moreno, C. E. y P. Rodríguez. 2010. A consistent terminology for quantifying species diversity? Oecologia 163:279-282.

Moreno, C. E. y P. Rodríguez. 2011. Commentary: Do we have a consistent terminology for species diversity? Back to basics and toward a unifying framework. Oecologia 167:889-892.

Nekola, J. C. y P. S. White. 1999. The distance decay of similarity in biogeography and ecology. Journal of Biogeography
26:867-878.

Novotny, V. y G. D. Weiblen. 2005. From communities to continents: beta diversity of herbivorous insects. Annales Zoologici Fennici 42:463-475.

Pélissier, R., P. Couteron y S. Dray. 2008. Analyzing or explaining beta diversity? Comment. Ecology 89:32273232 .

Preston, F.W. 1960. Time and space and the variation of species. Ecology 41:611-627.

Qian, H. y R. E. Ricklefs. 2007. A latitudinal gradient in largescale beta diversity for vascular plants in North America. Ecology Letters 10:737-744.

Ricotta, C. y S. Burrascano. 2008. Beta diversity for functional ecology. Preslia 80:61-71.

Rodríguez, P. y H. T. Arita. 2004. Beta diversity and latitude in North American mammals: testing the hypothesis of covariation. Ecography 27:547-556.

Rosenzweig, M. L. 1995. Species diversity in space and time. Cambridge University Press. 435 p.

Scheiner, S. M., A. Chiarucci., G. A. Fox., M. R. Helmus., D. J. McGlinn y M. R. Willig. 2011. The underpinnings of the relationship of species richness with space and time. Ecological Monographs 81:195-213.

Soininen, J., R. McDonald y H. Hillebrand. 2007. The distance decay of similarity in ecological communities. Ecography 30:3-12.

Tuomisto, H. y K. Ruokolainen. 2006. Analyzing or explaining beta diversity? Understanding the targets of different methods of analysis. Reply. Ecology 87:2697-2708.

Tuomisto, H. y K. Ruokolainen. 2008. Analyzing or explaining beta diversity? Reply. Ecology 89:3244-3256.

Tuomisto, H. 2010a. A diversity of beta diversities: straightening up a concept gone awry. Part 1 . Defining beta diversity as a function of alpha and gamma diversity. Ecography 33:2-22.

Tuomisto, H. 2010b. A diversity of beta diversities: straightening up a concept gone awry. Part 2. Quantifying beta diversity and related phenomena. Ecography 33:23-45.

Tuomisto, H. 2010c. A consistent terminology for quantifying species diversity? Yes it does exist. Oecologia 164:853-860.

Tuomisto, H. 2011. Do we have a consistent terminology for species diversity? Yes, if we choose to use it. Oecologia. Oecologia 167:903-911.

Ulrich, D. H., M. Almeida-Neto y N. J. Gotelli. 2009. A consumer's guide to nestedness analysis. Oikos 118:3-17.

Veech, J. A., K. S. Sumerville, T. O. Crist y J. C. Gering. 2002. The additive partitioning of species diversity: recent revival of an old idea. Oikos 99:3-9.

Veech, J. A. y T. O. Crist. 2010. Toward a unified view of diversity partitioning. Ecology 9:1988-1992.

Vellend, M. 2001. Do commonly used indices of $\beta$-diversity measure species turnover? Journal of Vegetation Science $12: 545-552$ 
Vellend, M., W. K. Cornwell, K. Magnuson-Ford y A. Mooers. 2011. Measuring phylogenetic biodiversity. In Biological diversity: frontiers in measurement and assessment, A. Magurran y B. J. McGill (eds.). Oxford University Press, New York. p. 194-207.

Westoby, M. 1993. Biodiversity in Australia compared with other continents. In Species diversity in ecological communities: historical and geographical perspectives, R. E. Ricklefts y D. Schluter (eds.). University of Chicago Press, Illinois. p. 170-177.

Whittaker, R. H. 1956. Vegetation of the Great Smoky Mountains. Ecological Monographs 26:1-80.

Whittaker, R. H. 1960. Vegetation of the Siskiyou Mountains, Oregon and California. Ecological Monographs 30:279-
338.

Whittaker, R. H. 1972. Evolution and measurement of species diversity. Taxon 12:213-251.

Whittaker, R. H. 1977. Evolution of species diversity in land communities. In Evolutionary biology, M. K. Hecht, W. C. Steere y B. Wallace (eds.). Plenum, New York. p.1-67.

Wright, D. H. y J. Reeves. 1992. On the meaning and measurement of nestedness of species assemblages. Oecologia 92:416-428.

Wright, D. H., B. D. Patterson, G. M. Mikkelson, A. Cutler y W. Atmar. 1998. A comparative analysis of nested subset patterns of species composition. Oecologia 113:1-20.

Wilson, M. V. y A. Shmida. 1984. Measuring beta diversity with presence-absence data. Journal of Ecology 72:1055-1064. 